\title{
Evidence of local structural order and spin-lattice coupling in the frustrated pyrochlore $\mathrm{Y}_{2} \mathrm{Ru}_{2} \mathrm{O}_{7}$
}

\author{
C. Castellano, ${ }^{1, *}$ G. Berti, ${ }^{2}$ S. Sanna, ${ }^{3}$ R. Ruiz-Bustos,${ }^{4}$ J. van Duijn, ${ }^{5}$ A. Brambilla, ${ }^{2}$ Á. Muñoz-Noval, ${ }^{6}$ \\ P. Carretta, ${ }^{3}$ L. Duò, ${ }^{2}$ and F. Demartin ${ }^{1}$ \\ ${ }^{1}$ Dipartimento di Chimica, Università degli Studi di Milano, via Golgi 19, 20133 Milano, Italy \\ ${ }^{2}$ Dipartimento di Fisica, Politecnico di Milano, piazza Leonardo da Vinci 32, 20133 Milano, Italy \\ ${ }^{3}$ Dipartimento di Fisica and Consorzio Nazionale Interuniversitario per le Scienze Fisiche della Materia (CNISM), Università di Pavia, \\ viale Bassi 6, 27100 Pavia, Italy \\ ${ }^{4}$ Departamento de Mecánica, Universidad de Córdoba, Córdoba, 14071, Spain \\ ${ }^{5}$ Instituto de Investigación en Energías Renovables, Departamento de Física Aplicada,Universidad de Castilla-La Mancha, \\ Albacete, 02006, Spain \\ ${ }^{6}$ Instituto de Ciencia de Materiales de Madrid-Consejo Superior de Investigaciones Cientificas (ICMM-CSIC) and Spanish Collaborating \\ Research Group (CRG), European Synchrotron Radiation Facility (ESRF), 71 Avenue des Martyrs, 38000 Grenoble, France
}

(Received 23 February 2015; revised manuscript received 19 May 2015; published 8 June 2015)

\begin{abstract}
We present an extended $\mathrm{x}$-ray absorption fine structure study of the pyrochlore $\mathrm{Y}_{2} \mathrm{Ru}_{2} \mathrm{O}_{7}(8-298 \mathrm{~K})$. We find evidence, on a local scale, of a significant magnetoelastic coupling at the Néel temperature $T_{\mathrm{N}} \sim 77 \mathrm{~K}$ pointed out by a huge Debye-Waller $\sigma^{2}$ factor deviation from a correlated temperature dependent Debye-like local order behavior plus a temperature independent static contribution. Moreover, we notice the occurrence of a potential local order-disorder structural phase transition at $T^{*}=150 \mathrm{~K}$. This anomalous behavior is consistent with the pyrochlore's predisposition towards structural disorder and with a strong spin-phonon correlation. Remarkably the low-temperature order competes with the tendency of magnetic frustration to induce a less symmetric local structure.
\end{abstract}

DOI: 10.1103/PhysRevB.91.224101

\section{INTRODUCTION}

Pyrochlore oxides offer a valuable playground to investigate the interplay between magnetic competitions and geometric frustration of the lattice structure. These systems have a general chemical formula $A_{2} B_{2} \mathrm{O}(1)_{6} \mathrm{O}(2)$, where $A$ is a trivalent (or divalent) rare earth or $\mathrm{Y}$ ion and $B$ is a tetravalent (or pentavalent) transition metal ion. Differently from the closely related fluorite $\left(A X_{2}\right)$ structure, in pyrochlores $1 / 8$ of the anions are absent (oxygen vacancy at the $8 a$ Wyckoff site). Both the $A$ and $B$ sites form an interpenetrating network of corner-sharing polyhedra (see Fig. 1 drawn using the VESTA 3 software [1]). When populated by magnetic ions with competing interactions, these materials can display novel shortrange ordered phases characteristic of geometrically frustrated magnets such as spin glasses, spin ices, and spin liquids [2]. Due to nearly infinite possibilities for site substitution and vacancies, pyrochlores can be employed as insulators, ion and electron conductors, superconductors, catalysts, robust nuclear waste forms, ion exchangers, phosphors, and solid-oxide fuel cell (SOFC) cathodes $[3,4]$.

It is important to note that within the pyrochlore structure, the composition-dependent $\mathrm{O}(1)$ positional parameter $x$ defines the polyhedral distortion and structural deviation from the ideal structure. For $x(\mathrm{O} 1)=0.3125$, the $B$ site is a regular octahedron and the $A$ site is a distorted trigonal scalenohedron (ideal pyrochlore or ordered structure), while for $x(\mathrm{O} 1)=$ 0.3750 , the $A$-site coordination polyhedron is a regular cube and the $B$-site polyhedron is distorted to a trigonally flattened octahedron (topology of the so-called defect or disordered fluorite structure) $[2,3]$. In the $x$ range $0.320-0.345$, the $B \mathrm{O}(1)_{6}$

\footnotetext{
*Corresponding author: carlo.castellano@unimi.it
}

PACS number(s): 75.47.Lx, 61.05.cj, 75.30.Cr, 81.30.Hd

octahedron is always slightly distorted at room temperature with the $\mathrm{O}(1)-B-\mathrm{O}(1)$ angle between $81^{\circ}$ and $100^{\circ}\left(90^{\circ}\right.$ for a regular octahedron). The $\mathrm{O}(1) x$ coordinate, therefore, actually determines the degree of order of the structure, and at high temperatures some of the pyrochlore phases undergo an order-disorder transition changing to the disordered fluorite phase [5].

Within this family of materials, the ruthenium pyrochlores ( $B=\mathrm{Ru}$ ) display a multitude of interesting properties because of their high chemical and structural flexibility. These properties include metal-insulator transition and long-range ordering as well as spin-singlet transitions [6-10]. As such they are also useful for specific applications. The wide variety of ground states is due to the fact that $\mathrm{Ru}$ can be either in the $4+$ or $5+$ oxidation state, promoting good electrical and catalytic properties. As a consequence, a very good electrochemical behavior of Ru-based pyrochlores has been observed at temperatures as low as $350^{\circ} \mathrm{C}$, which has motivated their study as intermediate temperature SOFC (IT-SOFC) cathodes [11].

Within the ruthenium pyrochlores, $\mathrm{Y}_{2} \mathrm{Ru}_{2} \mathrm{O}_{7}$ is characterized by rather large antiferromagnetic exchange couplings $J$ among $\mathrm{Ru}^{4+} S=1$ spins, giving rise to a Curie-Weiss temperature of about $1100 \mathrm{~K}$ and eventually an antiferromagnetic phase transition at $T_{\mathrm{N}} \sim 77 \mathrm{~K}$ [6]. Band structure calculations reveal $\mathrm{Y}_{2} \mathrm{Ru}_{2} \mathrm{O}_{7}$ to be a Mott-Hubbard insulator, and the spins can be considered localized at the corners of regular tetrahedra, as in many frustrated magnets with the pyrochlore structure [7]. Magnetic susceptibility measurements show a large zero field cooled (ZFC)-field cooled (FC) deviation, suggesting the presence of a spin-glass-like behavior, while neutron scattering experiments show long-range magnetic order of the ruthenium moments [12]. The ordered moment $\left(\mu_{\text {ord }} \sim 1.36 \mu_{B}\right)$, as probed by neutrons, is however, significantly reduced compared to the effective moment $\left[\mu_{\text {eff }} \sim 3.1(1) \mu_{B}\right]$ evaluated 

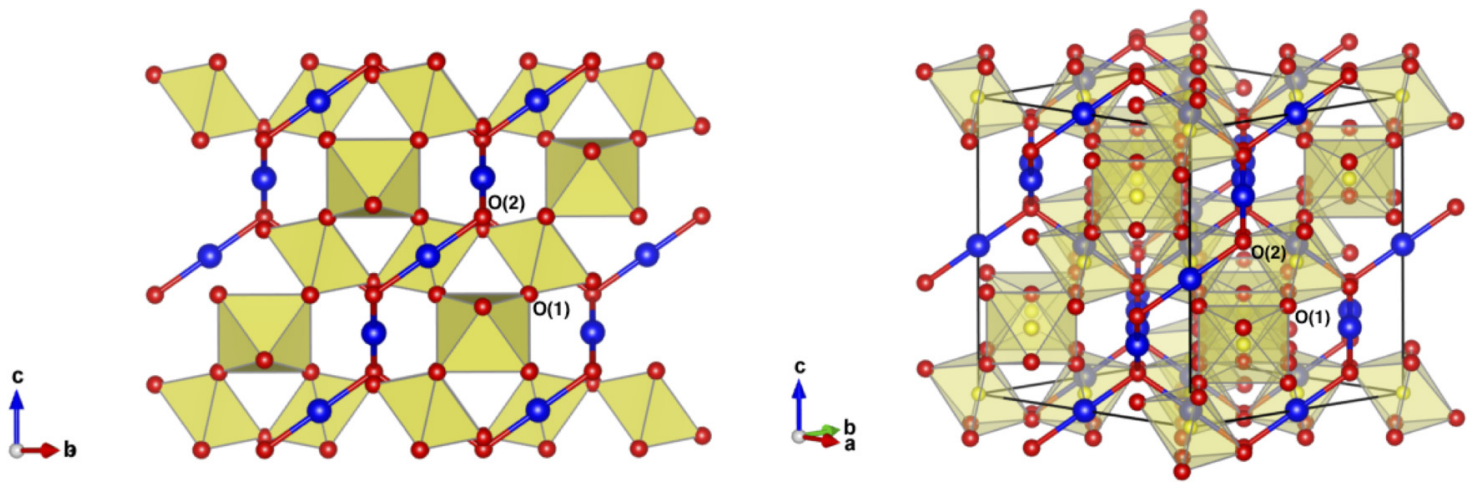

FIG. 1. (Color online) Perspective views of the $\mathrm{Y}_{2} \mathrm{Ru}_{2} \mathrm{O}_{7}$ crystal structure (space group $F d-3 m$ ). Y: blue spheres (Wyckoff site $16 d$ ); Ru atoms lie in the center of the yellow octahedra (Wyckoff site 16c); O: red spheres \{Wyckoff sites $48 f[\mathrm{O}(1)$, at the vertex of the octahedra] and $8 b[\mathrm{O}(2)$, bridging $\mathrm{Y}$ atoms $]\}$.

by magnetization measurements [12,13]. More recent muon spin relaxation measurements have clearly shown a long-range ordering throughout the bulk, hence excluding a glassy or short-range ordered ground state and supporting that $\mathrm{Y}_{2} \mathrm{Ru}_{2} \mathrm{O}_{7}$ undergoes antiferromagnetic ordering below $T_{\mathrm{N}}$ [14].

While various diffraction studies confirm the cubic pyrochlore structure above and below the Néel temperature, recent infrared (IR) and Raman measurements show anomalies in various phonon modes at $T_{\mathrm{N}}[15,16]$. These measurements suggest that strong spin-phonon coupling effects might play an important role in allowing the transition to a long-range ordered state to occur. This is consistent with inelastic neutron scattering measurements, which revealed that at $T_{\mathrm{N}}$ $\mathrm{Y}_{2} \mathrm{Ru}_{2} \mathrm{O}_{7}$ undergoes a "strong coupling" transition where potential magnetoelastic effects allow a spin-Peierls type phase transition [17]. The frustrated interactions, combined with the lattice coupling, are thought to result in the formation of a low-energy manifold of near-degenerate states with a characteristic interatomic "form factor." The exact nature of the spin-phonon coupling or its importance are, however, not yet clearly understood.

In this context, the aim of the present paper is to characterize and understand in greater detail the above described magnetostructural coupling by local structure information available from extended $\mathrm{x}$-ray absorption fine structure (EXAFS) data, able to go beyond the medium-long diffraction range. Indeed, whereas powder diffraction experiments on $\mathrm{Y}_{2} \mathrm{Ru}_{2} \mathrm{O}_{7}[x(O 1)=0.33536(3)]$ show the presence of six $\mathrm{Ru}-\mathrm{O}(1)$ identical interatomic distances $[R=1.9911(1) \AA]$, a Ru-O(1)-Ru bond angle of $128.5^{\circ}$, six $\mathrm{O}(1)-\mathrm{Ru}-\mathrm{O}(1)$ bond angles of $81.47^{\circ}$ and two of $98.53^{\circ}[2,18]$, no EXAFS study for this composition has previously been published. In the following, we will show that the EXAFS results here reported are quite remarkable as they present a direct experimental evidence of a huge magnetoelastic coupling at $T_{\mathrm{N}} \sim 77 \mathrm{~K}$. In addition, at a critical temperature $T^{*}$ of about $150 \mathrm{~K}(\sim 2 \times$ $T_{\mathrm{N}}$ ), an unexpected local structure distortion of the $\mathrm{Ru}-\mathrm{Ru}$ and $\mathrm{Ru}-\mathrm{Y}$ second coordination shell and of the $\mathrm{RuO}(1)_{6}$ first shell octahedra to a more ordered state seems to occur.

The obtained results are consistent with the pyrochlore predisposition towards disorder and structural distortion and with a strong correlation between spin and phonon degrees of freedom. Nonetheless, the observed tendency of $\mathrm{Y}_{2} \mathrm{Ru}_{2} \mathrm{O}_{7}$ towards a low-temperature ordered structure appears to compete with the "normal" tendency of pyrochlores to relieve frustration through a lattice distortion. It might however be a precursor for spin-lattice coupling and the subsequent transition to a long-range ordered state to occur.

\section{EXPERIMENTAL DETAILS AND DATA ANALYSIS}

A powdered sample of $\mathrm{Y}_{2} \mathrm{Ru}_{2} \mathrm{O}_{7}$ was prepared using the solid state reaction method. Mixtures of $\mathrm{Y}_{2} \mathrm{O}_{3}$ and $\mathrm{RuO}_{2}$ in proper molar ratio were intimately mixed, pressed into pellets, and reacted at $1000-1200^{\circ} \mathrm{C}$ in air with intermediate grindings. The sample was revealed to be single phase by $\mathrm{X}$-ray powder diffraction performed using a Philips XPert Pro diffractometer with a copper source and incident beam monochromator. Rietveld analysis confirmed the cubic pyrochlore structure.

The EXAFS measurements were carried out at the $\mathrm{Ru}$ $K$-edge $(22117 \mathrm{eV})$ in transmission geometry at the European Synchrotron Radiation Facility (ESRF) on beamline BM25A, using a double $\mathrm{Si}(111)$ crystal monochromator of the pseudo channel-cut type refrigerated at $200 \mathrm{~K}$ by a homemade ethanol cooling system. The energy resolution was close to $1.2 \mathrm{eV}$.

The polycrystalline $\mathrm{Y}_{2} \mathrm{Ru}_{2} \mathrm{O}_{7}$ pyrochlores were dispersed in a cellulose matrix and pressed into pellets, optimizing the jump at the absorption edge. In order to accurately follow the behavior of the local order and structure parameters as a function of temperature, we measured accurate spectra at 26 different temperatures between 8 and $298 \mathrm{~K}$, thickening the points in the 40-85 $\mathrm{K}$ range (since the magnetic ordering transition occurs at $T_{\mathrm{N}} \sim 77 \mathrm{~K}$ ). An Oxford CV-F optistat liquid helium (LHe) cryostat with a LHe Dewar supply was used. At least two scans were collected at each temperature for averaging and to check reproducibility. The EXAFS data were reduced using the Demeter package standard procedures [19]; fits of the $k^{3}$ weighted EXAFS data were carried out in $r$ space using theoretical functions from the FEFF9 code [20]. Examples of the resulting high-quality $k$-space data at several temperatures and up to $16 \AA^{-1}$ are shown in Fig. 2 .

To include the first and further neighbors in the data (full fit range: $1.05-3.80 \AA$ ), we used the first shell $\mathrm{Ru}-\mathrm{O}(1)$ peak with $R=1.99 \AA$ bond length and coordination number $N_{\mathrm{Ru}-\mathrm{O}}=6$, 


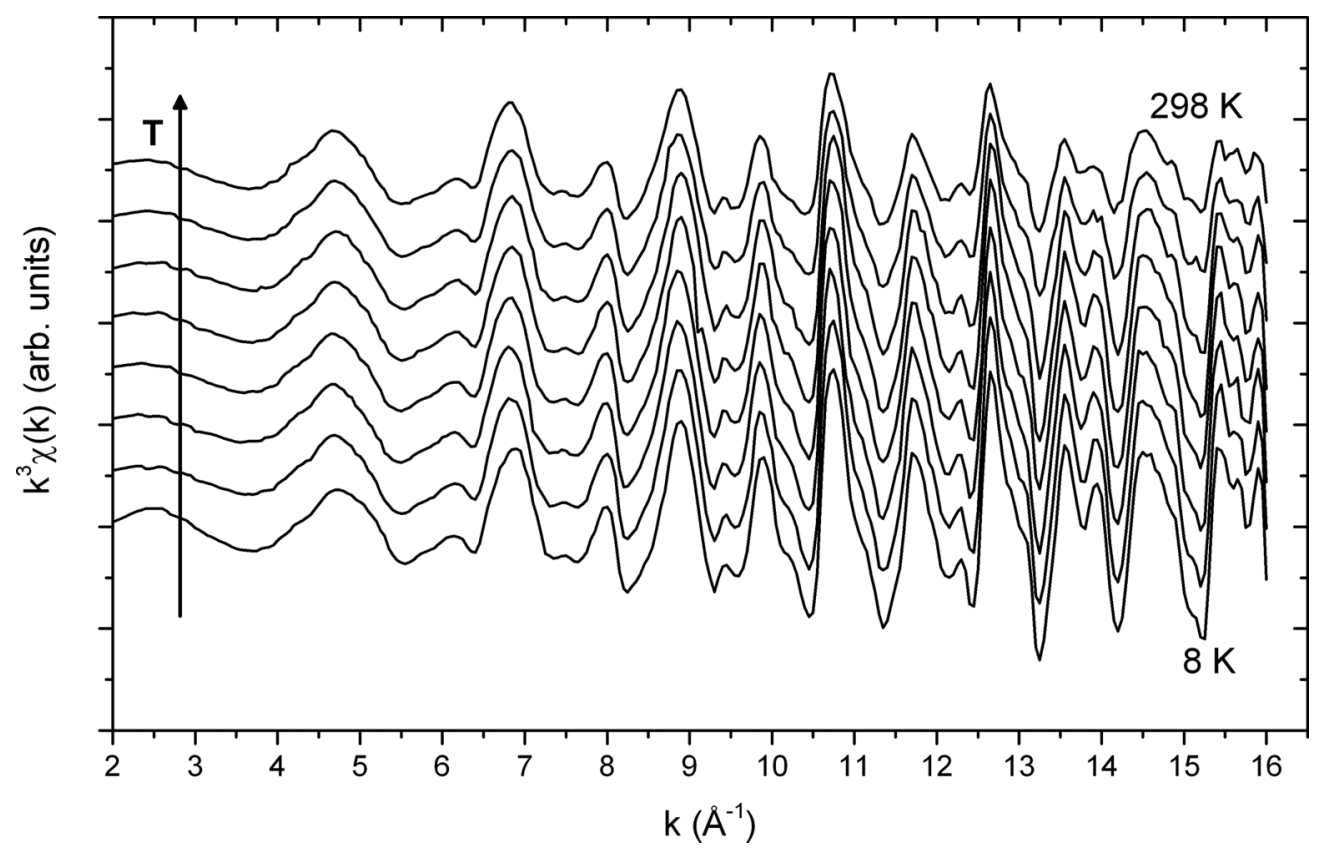

FIG. 2. $k^{3} \chi$ (k) EXAFS signals at different temperatures between $8 \mathrm{~K}$ (lower curve) and $298 \mathrm{~K}$ (upper curve). Data have been offset for clarity.

at $298 \mathrm{~K}$, the $\mathrm{Ru}-\mathrm{Ru}$ and $\mathrm{Ru}-\mathrm{Y}$ second shell peaks $(N=6$ each), two other longer Ru-O(1) peaks ( $N=6$ and 12 , respectively) and two multiscattering peaks. These last two peaks included in the fit were the triple forward scattering through the absorber $\mathrm{Ru}-\mathrm{O}(1)-\mathrm{Ru}-\mathrm{O}(1)(N=6)$ and the double scattering $\mathrm{Ru}-\mathrm{O}(1)-\mathrm{O}(1)(N=24)$. Alternatively, two first subshells Ru-O(1) (with coordination numbers $N_{\text {shorter }}=4, N_{\text {longer }}=2$ and $R_{\text {shorter }}=2.00 \AA, R_{\text {longer }}=2.05 \AA$, respectively, still at $298 \mathrm{~K}$ ) were used.

An overview of all the different paths used in our fits is shown in Fig. 3.
The initial fitting parameters were the Debye-Waller factors $\sigma^{2}$, the interatomic bond lengths $R$, the $E_{0}$ shift in the edge energy with respect to the theoretical value, and the amplitude reduction factor $S_{0}^{2}$ from multielectron effects. This last factor is generally between 0.8 and 1.0. At low $T$, the lattice is well ordered, and $S_{0}^{2}$ was determined from the average of several fits to scans at $8 \mathrm{~K}$ and fixed at 0.96 . The $E_{0}$ shift was also constrained to a single value for all the paths. In order to have fewer independent parameters and to limit the correlations among them, the Debye-Waller factors of the two subshells were linked to be equal to one another, and the interatomic

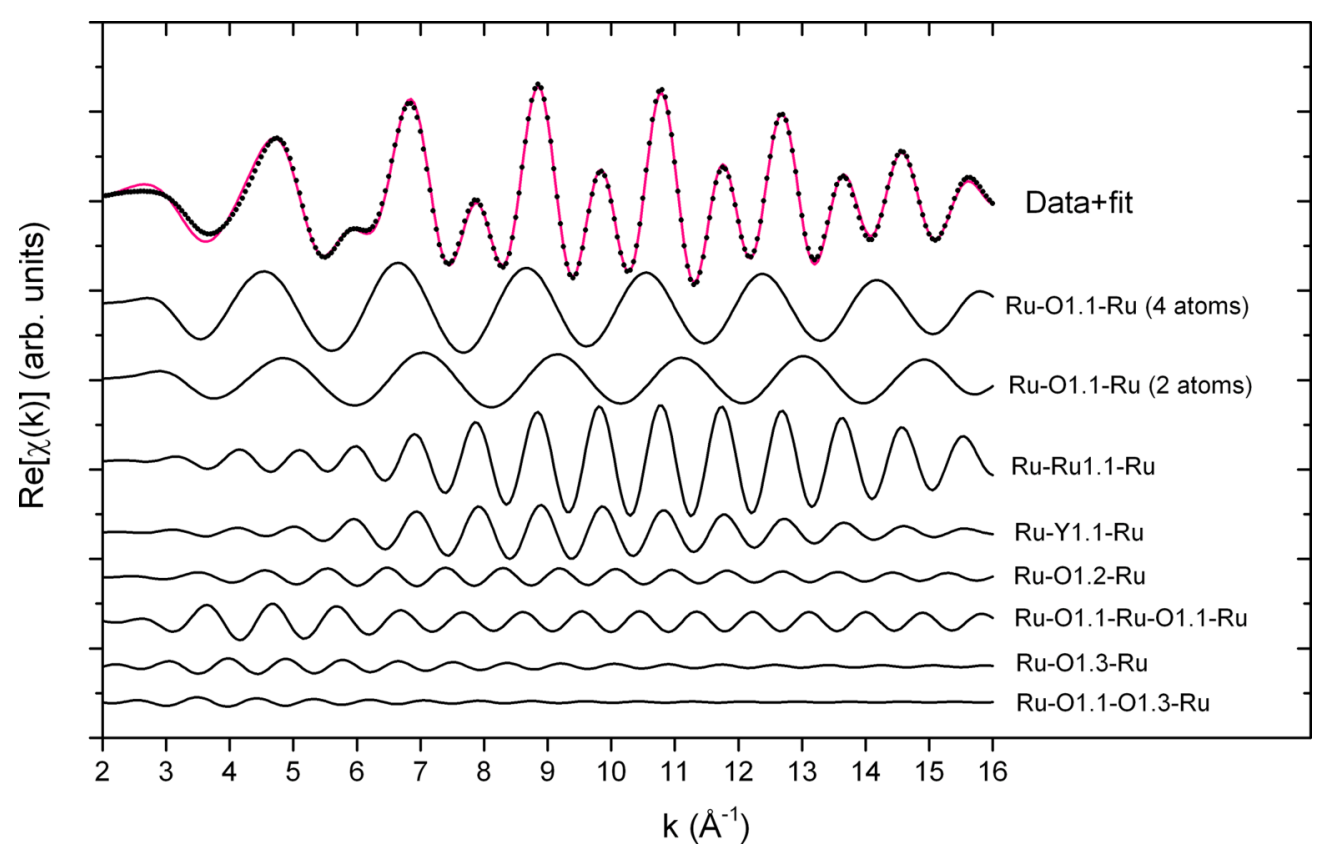

FIG. 3. (Color online) Overview of all the distinct contributions to the fits at $298 \mathrm{~K}$. Data have been offset for clarity. 
distances of the two longer $\mathrm{Ru}-\mathrm{O}(1)$ single-scattering and of the two multiscattering peaks were constrained to the structure [18]. The coordination numbers were fixed to the previously reported values for the first shell and to the crystallographic values for all the other shells and paths employed in the fit. In this way, the uncertainty on the Debye-Waller factors $\sigma^{2}$ was reduced [21]. Therefore, the final number of fit parameters was 11 and 12 in the one subshell and in the two subshell models, respectively, well below the number of independent data points, 25, estimated from Stern's criteria [22]. The parameters to be varied were the remaining unconstrained $\sigma^{2}$, $R$, and a single $E_{0}$ shift.

\section{RESULTS AND DISCUSSION}

In Fig. 4 (top panel), the temperature dependence of the magnetic susceptibility is displayed in both $\mathrm{ZFC}$ and $\mathrm{FC}$ conditions. The antiferromagnetic phase transition at $T_{\mathrm{N}} \sim$ $77 \mathrm{~K}$ is shown according to the literature $[6,12]$. In Fig. 4 (bottom panel), the local order or Debye-Waller factor $\sigma^{2}$

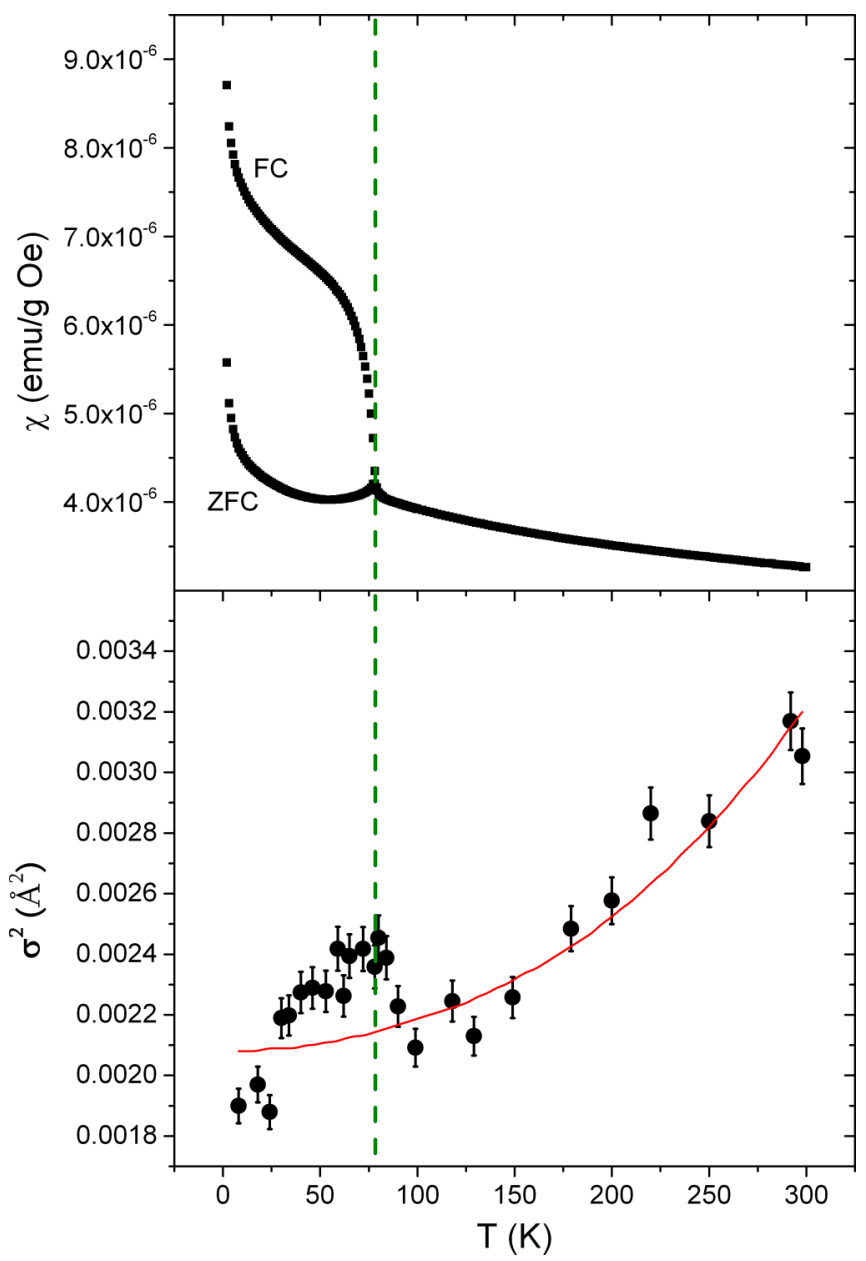

FIG. 4. (Color online) (Top) Temperature dependence of the magnetic susceptibility in both ZFC and FC conditions with an external applied field $\mu_{0} \mathrm{H}=0.1$ Tesla; (bottom) $\mathrm{Ru}-\mathrm{O}$ (1) first shell $\sigma^{2}\left(\AA^{2}\right)$ as a function of temperature (the green dashed line indicates $T_{\mathrm{N}}$ and the continuous red line is the fit to a correlated Debye model plus a static offset). for the $\mathrm{Ru}-\mathrm{O}(1)$ first coordination shell obtained from the fit performed with one subshell is reported as a function of temperature. A clear peak appears in the temperature region around the antiferromagnetic transition, indicating the occurrence of a magnetoelastic coupling that we point out as an additional local disorder. Starting from room temperature, the local disorder $\sigma^{2}$ decreases until $100 \mathrm{~K}$ and after that point increases reaching a maximum at about $78 \mathrm{~K}$; this last temperature coincides within the experimental error with the magnetic transition $T_{\mathrm{N}}$ (see Fig. 4, top panel). It is worth noticing that this anomalous $\sigma^{2}$ peak is not only huge in amplitude but covers also a very broad temperature range, roughly from 100 to $30 \mathrm{~K}$. The obtained result is even more significant since in the literature neither a dynamic disorder nor a coupling between a magnetic and a structural transition were observed in these compounds by other structural techniques such as diffraction [13]. On the other hand, the reported anomalies in the temperature dependence of one Raman-active mode and of six phonon modes indicate a possible spin-phonon coupling that could be related to what we are seeing, but in a less direct way $[15,16]$.

In general, the $\sigma^{2}$ parameter follows a temperature dependent correlated Debye-like behavior $\sigma_{D}{ }^{2}$ at high temperatures, with a correlated Debye temperature $\theta_{\mathrm{D}}$ around $800 \mathrm{~K}$ in systems like these, plus a temperature independent static contribution $\sigma_{S}^{2}$ due to the presence of a possible distorted environment. The Debye-Waller factor shown in Fig. 4 was fitted above $100 \mathrm{~K}$ considering $\sigma^{2}(T)=\sigma_{D}{ }^{2}+\sigma_{S}{ }^{2}$, as already shown in previous papers [21,23], where the temperature dependent dynamic contribution $\sigma_{D}{ }^{2}$ plus a static offset is usually a good approximation for all the phonon modes [24,25]. The obtained fit parameters values were the correlated Debye temperature $\theta_{\mathrm{D}}=831 \pm 26 \mathrm{~K}$, which is a measure of the $\mathrm{Ru}-\mathrm{O}$ bond strength, in accordance with other pyrochlores [26], and the static offset $\sigma_{S}^{2}=-0.0002(3) \AA^{2}$. This negative value is within the estimated uncertainty, and it is consistent with zero local distortions at the lowest temperatures. In Fig. 4 (bottom panel), the huge $\sigma^{2}$ deviation from the correlated Debye-like local order fit (continuous line) is clearly shown. A magnetoelastic coupling interpretation related to such behaviors was already suggested in other complex oxides like manganites and ruthenocuprates [21,23].

The Debye-Waller factors $\sigma^{2}(T)$ of the second shell peaks $\mathrm{Ru}-\mathrm{Ru}$ and $\mathrm{Ru}-\mathrm{Y}$ have a monotonic behavior and do not show any anomaly at about $77 \mathrm{~K}$ in the one subshell fit, indicating that most likely the oxygens are mainly involved in the magnetoelastic coupling.

We hypothesize that the observed additional local disorder may be associated with the presence of a bimodal distribution of the $\mathrm{Ru}-\mathrm{O}$ interatomic distances for the first shell $\mathrm{RuO}(1)_{6}$ octahedra according to the described two subshell fit model. This last model was selected considering the different electronegativity of the second neighbors, ruthenium and yttrium, taking into account the aforementioned tendency to octahedral distortion and, compared to alternative models tested, was supported by the obtained best fit residuals.

We have to recall here that the analysis performed with a two subshell model has a widely argued limit determined by the EXAFS resolving power between two scattering shells involving the same chemical species at different distances. 


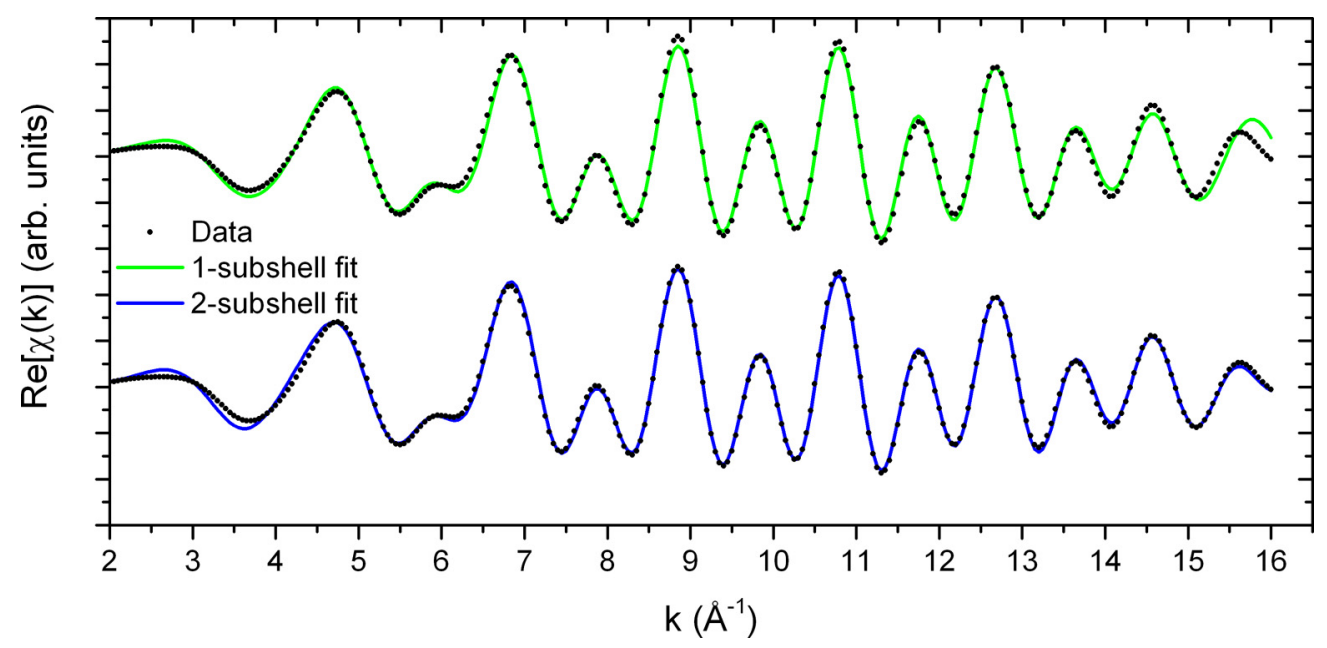

FIG. 5. (Color online) Back Fourier transform best-fit curves obtained considering one (upper curves) or two (lower curves) Ru-O subshells at $298 \mathrm{~K}$. The two fit curves have been shifted for clarity.

Since the maximum $k$ vector for our data is $k_{\max }=16 \AA^{-1}$, we can accurately resolve splittings $\Delta R>\pi / 2 k_{\max } \sim 0.098 \AA$ $[27,28]$. Therefore it needs to be treated with care even if no other technique apart from EXAFS allows one to obtain on a local scale similar results that, in our opinion, deserve to be reported in the light of the above cited physical reasons related to structural distortion.

In Fig. 5, typical back Fourier transform fits of $k^{3}$-weighted filtered data at $298 \mathrm{~K}$, performed with one or two Ru-O(1) distances, are shown [21]. In this framework, the quality of the two fits was checked by means of the residual $\rho=$ $\Sigma_{i} k_{i}^{3}\left[\chi_{\exp }\left(k_{i}\right)-\chi_{\text {theo }}\left(k_{i}\right)\right]^{2} / \Sigma_{i} k_{i}^{3}\left[\chi_{\exp }\left(k_{i}\right)\right]^{2}$, where $\chi_{\exp }\left(k_{i}\right)$ and $\chi_{\text {theo }}\left(k_{i}\right)$ are the experimental and the calculated EXAFS signals at the wave vector $k_{i}$. The Ru-O(1) bimodal distribution fit gives a mean residual of $1.5 \%$ (calculated from $1.0 \%$ and $1.9 \%$ in the full $T$ range), about $30-35 \%$ lower than that obtained by the analysis performed with one subshell at all the temperatures $[21,23,29]$.

The effect observed for the two main second coordination shell peaks Ru-Ru and Ru-Y, shown in Fig. 6 (upper part), is fairly more reliable due to the involvement of different backscattering chemical species. We observe a sudden increase of the six Ru-Ru bond lengths and a simultaneous decrease of the six Ru-Y interatomic distances just above $T^{*}=150 \mathrm{~K}$, with a splitting of the two peaks of about $0.080 \AA$; below $T^{*}$ they tend to have the same value inside the standard deviation of $0.005 \AA$, as found by neutron diffraction [18]. This behavior seems to reveal a characteristic temperature where a transition like a local order-disorder changeover occurs.

On the other hand, the first shell $\mathrm{Ru}-\mathrm{O}(1)$ interatomic distances obtained from the two subshell model are reported in the lower part of Fig. 6. We observe that at temperatures up to about $T^{*}=150 \mathrm{~K}$, the one and two bond length distributions coincide within the statistical error, whereas at higher temperatures the two longer $R$ apical values suddenly increase and the four shorter basal ones remain the same as at lower $T$. The maximum observed splitting of the first shell $\mathrm{Ru}-\mathrm{O}(1)$ distance is about $0.045 \AA$, beyond the above cited EXAFS ability to resolve split peaks. Therefore, we can say that a distortion larger than the standard deviation or uncertainty on the single $R$ value is revealed but also that it cannot be strictly stated due to the intrinsic resolution limit of the technique.

However, a local structure distortion like this is certainly much more consistent with the predisposition towards disorder and octahedron distortion characteristic of pyrochlores [30] than the six identical interatomic distances observed by diffraction [18]. Indeed, as a further demonstration of this

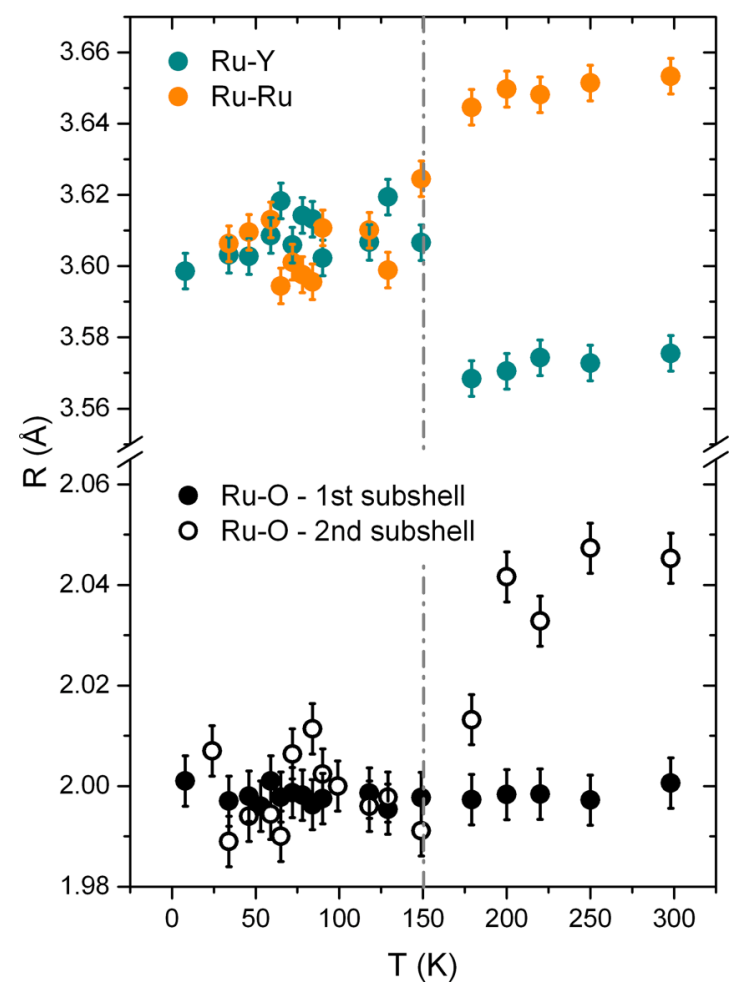

FIG. 6. (Color online) (Upper) Second shell Ru-Y and Ru-Ru bond lengths, as a function of temperature; (lower) bimodal distribution of the $\mathrm{Ru}-\mathrm{O}$ bond lengths $\left[N_{\mathrm{Ru}-\mathrm{O}}=4\right.$ and $N_{\mathrm{Ru}-\mathrm{O}}=2$ for the shorter basal (solid circles) and longer apical (open circles) interatomic distances]. The dashed line indicates the transition temperature at $T^{*}=150 \mathrm{~K}$. 
aptitude, in related $A$-site doped systems like $\mathrm{Bi}_{2-x} \mathrm{Y}_{\mathrm{x}} \mathrm{Ru}_{2} \mathrm{O}_{7}$, with increasing the smaller yttrium ion amount, the $\mathrm{Ru}-$ $\mathrm{O}(1)-\mathrm{Ru}$ bond angle decreases, the $\mathrm{Ru}-\mathrm{O}(1)$ interatomic distance increases, the bend in the $\mathrm{RuO}(1)_{6}$ corner shared zig-zag chains, and consequently the octahedron distortion also increases [31].

Still following this working hypothesis, the $\mathrm{RuO}(1)_{6}$ octahedron distorted at high temperatures, whereas the local structure transition suggested at about $T^{*}$ could be a precursor effect of the magnetoelastic coupling observed at the lower temperatures as a broad $\sigma^{2}$ peak (Fig. 4). This feature is also in agreement with the occurrence of short-range ordered phases such as spin glass and spin ice in several geometrically frustrated pyrochlore compositions $[13,32]$. This tendency of pyrochlores towards a low-temperature ordered structure appears to compete with the tendency of the material to relieve frustration through a lattice distortion. In fact, in frustrated magnets one typically observes at $T^{*}<J$ a distortion to a lower symmetry phase and eventually at $T_{\mathrm{N}}$ the system collapses in a well-defined ground state $[33,34]$. Here, in spite of the significant spin-lattice coupling, the structural changes appear not to be driven by frustration but could be a prerequisite for the transition to a long-range ordered state to occur.

\section{CONCLUSIONS}

Summarizing, EXAFS provides important information (that cannot be obtained by traditional $\mathrm{x}$-ray or neutron diffraction techniques) for the first and second neighbor shells by showing a huge magnetoelastic coupling on the local scale with the lack of a frustration driven distortion at $T_{\mathrm{N}}$.

An additional local order-disorder phase transition at a critical temperature $T^{*}=150 \mathrm{~K}$ is not definitely ascertained but is in agreement with the tendency of pyrochlores to structurally distort. To our knowledge, there is no other way to evidence this local structure effect. We believe that our findings will allow additional physical and structural insights related to the order-disorder and magnetostructural behaviors of these strongly correlated electron systems, as well as critiques of and reflections on their local order and structure.
[1] K. Momma and F. Izumi, J. Appl. Crystallogr. 44, 1272 (2011).

[2] J. S. Gardner, M. J. P. Gingras, and J. E. Greedan, Rev. Mod. Phys. 82, 53 (2010).

[3] R. C. Ewing, W. J. Weber, and J. Lian, J. Appl. Phys. 95, 5949 (2004).

[4] Mark T. Weller, R. W. Hughes, J. Rooke, C. S. Knee, and J. Reading, Dalton Trans. 3032 (2004).

[5] B. P. Mandal, A. Banerji, V. Sathe, S. K. Deb, and A. K. Tyagi, J. Solid State Chem. 180, 2643 (2007).

[6] S. Yoshii and M. Sato, J. Phys. Soc. Jpn. 68, 3034 (1999).

[7] K.-S. Lee, D.-K. Seo, and M.-H. Whangbo, J. Solid State Chem. 131, 405 (1997).

[8] M. K. Haas, R. J. Cava, M. Avdeev, and J. D. Jorgensen, Phys. Rev. B 66, 094429 (2002).

[9] S. Lee, J.-G. Park, D. T. Adroja, D. Khomskii, S. Streltsov, K. A. McEwen, H. Sakai, K. Yoshimura, V. I. Anisimov, D. Mori, R. Kanno, and R. Ibberson, Nat. Mater. 5, 471 (2006).

[10] A. Yamamoto, P. A. Sharma, Y. Okamoto, A. Nakao, H. A. Katori, S. Niitaka, D. Hashizume, and H. Takagi, J. Phys. Soc. Jpn. 76, 043703 (2007).

[11] J. B. Goodenough, R. Manoharan, and P. Paranthaman, J. Am. Chem. Soc. 112, 2076 (1990).

[12] M. Ito, Y. Yasui, M. Kanada, H. Harashina, S. Yoshii, K. Murata, M. Sato, H. Okumura, and K. Kakurai, J. Phys. Soc. Jpn. 69, 888 (2000).

[13] R. Kmieć, Ż. Świątkowska, J. Gurgul, M. Rams, A. Zarzycki, and K. Tomala, Phys. Rev. B 74, 104425 (2006).

[14] S. J. Blundell, T. Lancaster, P. J. Baker, W. Hayes, F. L. Pratt, T. Atake, D. S. Rana, and S. K. Malik, Phys. Rev. B 77, 094424 (2008).

[15] J. S. Bae, I.-S. Yang, J. S. Lee, T. W. Noh, T. Takeda, and R. Kanno, Phys. Rev. B 73, 052301 (2006).

[16] J. S. Lee, T. W. Noh, J. S. Bae, I.-S. Yang, T. Takeda, and R. Kanno, Phys. Rev. B 69, 214428 (2004).

[17] J. van Duijn, N. Hur, J. W. Taylor, Y. Qiu, Q. Z. Huang, S.-W. Cheong, C. Broholm, and T. G. Perring, Phys. Rev. B 77, 020405(R) (2008).
[18] B. J. Kennedy, Acta Crystallogr. Sect. C 51, 790 (1995).

[19] B. Ravel and M. Newville, J. Synchrotron Radiat. 12, 537 (2005).

[20] A. L. Ankudinov, B. Ravel, J. J. Rehr, and S. D. Conradson, Phys. Rev. B 58, 7565 (1998).

[21] C. Castellano, M. Ferretti, A. Martinelli, and M. R. Cimberle, J. Alloys Compd. 478, 479 (2009).

[22] E. A. Stern, Phys. Rev. B 48, 9825 (1993).

[23] A. Paolone, C. Castellano, O. Palumbo, F. Cordero, R. Cantelli, A. Vecchione, M. Gombos, S. Pace, and M. Ferretti, Physica C 467, 167 (2007).

[24] G. Beni and P. M. Platzmann, Phys. Rev. B 14, 1514 (1976).

[25] N. W. Ashcroft and N. D. Mermin, in Solid State Physics (Saunders College, Philadelphia, 1976).

[26] M. B. Johnson, D. D. James, A. Bourque, H. A. Dabkowska, B. D. Gaulin, and M. A. White, J. Solid State Chem. 182, 725 (2009).

[27] P. A. Lee, P. H. Citrin, P. Eisenberger, and B. M. Kincaid, Rev. Mod. Phys. 53, 769 (1981).

[28] T. Keiber, F. Bridges, and B. C. Sales, Phys. Rev. Lett. 111, 095504 (2013).

[29] A. Paolone, C. Castellano, R. Cantelli, G. Rousse, and C. Masquelier, Phys. Rev. B 68, 014108 (2003).

[30] P. E. R. Blanchard, R. Clements, B. J. Kennedy, C. D. Ling, E. Reynolds, M. Avdeev, A. P. J. Stampfl, Z. Zhang, and L.-Y. Jang, Inorg. Chem. 51, 13237 (2012).

[31] R. Kanno, Y. Takeda, T. Yamamoto, Y. Kawamoto, and O. Yamamoto, J. Solid State Chem. 102, 106 (1993).

[32] M. Ito, Y. Yasui, M. Kanada, H. Harashina, S. Yoshii, K. Murata, M. Sato, H. Okumura, and K. Kakurai, J. Phys. Chem. Solids 62, 337 (2001).

[33] O.Tchernyshyov, R. Moessner, and S. L. Sondhi, Phys. Rev. Lett. 88, 067203 (2002).

[34] P. Carretta, N. Papinutto, C. B. Azzoni, M. C. Mozzati, E. Pavarini, S. Gonthier, and P. Millet, Phys. Rev. B 66, 094420 (2002). 